\title{
Incorporating genomic signatures into surgical and medical decision-making for elderly glioblastoma patients
}

\author{
Chibawanye I. Ene, MD, PhD, ${ }^{1}$ Patrick J. Cimino, MD, PhD, ${ }^{2}$ Howard A. Fine, MD, ${ }^{3}$ and \\ Eric C. Holland, MD, PhD4
}

\begin{abstract}
1Department of Neurological Surgery, University of Washington School of Medicine; '2Department of Pathology, Division of Neuropathology, University of Washington School of Medicine, Seattle, Washington; ${ }^{3}$ Meyer Cancer Center, Division of NeuroOncology, Department of Neurology, NewYork-Presbyterian Hospital/Weill Cornell Medicine, New York, New York; and ${ }^{4}$ Human Biology Division, Fred Hutchinson Cancer Research Center, Seattle, Washington
\end{abstract}

\begin{abstract}
Glioblastoma (GBM) is the most common type of malignant primary brain tumor in adults. It is a uniformly fatal disease (median overall survival 16 months) even with aggressive resection and an adjuvant temozolomide-based chemoradiation regimen. Age remains an independent risk factor for a poor prognosis. Several factors contribute to the dismal outcomes in the elderly population with GBM, including poor baseline health status, differences in underlying genomic alterations, and variability in the surgical and medical management of this subpopulation. The latter arises from a lack of adequate representation of elderly patients in clinical trials, resulting in limited data on the response of this subpopulation to standard treatment. Results from retrospective and some prospective studies have indicated that resection of only contrast-enhancing lesions and administration of hypofractionated radiotherapy in combination with temozolomide are effective strategies for optimizing survival while maintaining baseline quality of life in elderly GBM patients; however, survival remains dismal relative to that in a younger cohort. Here, the authors present historical context for the current strategies used for the multimodal management (surgical and medical) of elderly patients with GBM. Furthermore, they provide insights into elderly GBM patient-specific genomic signatures such as isocitrate dehydrogenase 1/2 (IDH1/2) wildtype status, telomerase reverse transcriptase promoter (TERTp) mutations, and somatic copy number alterations including CDK4/MDM2 coamplification, which are becoming better understood and could be utilized in a clinical trial design and patient stratification to guide the development of more effective adjuvant therapies specifically for elderly GBM patients.
\end{abstract}

https://thejns.org/doi/abs/10.3171/2020.7.FOCUS20418

KEYWORDS glioblastoma; age; elderly; supratotal resection; gross-total resection; hypofractionated radiation therapy; temozolomide; somatic copy number alterations

$\mathrm{G}$ LIOBLASTOMa (GBM) is the most common and most aggressive primary brain tumor in adults. Standard of care entails maximum safe resection followed by concurrent temozolomide (TMZ)-based chemotherapy and radiotherapy, a regimen associated with a median survival of 16 months..$^{2}$ Although GBM is uniformly fatal across all demographics, age remains an independent risk factor for a poor prognosis in newly diagnosed GBM cases, regardless of baseline function, tumor size, or administration of the standard treatment regimen. 3,4
From 1977 to 2000, the median age at GBM diagnosis was 63 years, with diagnosis increasing at a rate of $2.3 \%$ per year during that period. ${ }^{5}$ According to the Central Brain Tumor Registry of the United States (CBTRUS), the median age at diagnosis of GBM from 2005 to 2009 was 64 years and from 2011 to 2015 it was 65 years. ${ }^{6,7}$ Unfortunately, the management of GBM in the elderly (i.e., age $\geq 65$ years) remains variable, with a tendency toward conservative approaches that are not evidence-based. ${ }^{8}$ Since elderly patients remain underrepresented in many GBM clinical trials, most current management strategies are ex-

ABBREVIATIONS BEV = bevacizumab; CNS = central nervous system; EOR = extent of resection; $G B M=$ glioblastoma; $G G N=$ German Glioma Network; $G T R=$ grosstotal resection; IDH1/2 = isocitrate dehydrogenase 1/2; MGMT = [6]-methylguanine-DNA methyltransferase; OS = overall survival; $P F S=$ progression-free survival; $P R=$ partial resection; RT = radiation therapy; SCNA = somatic copy number alteration; SRS = stereotactic radiosurgery; STR = subtotal resection; SupTR = supratotal resection; $T E R T p=$ telomerase reverse transcriptase promoter; TMZ = temozolomide; VEGF = vascular endothelial growth factor.

SUBMITTED May 21, 2020. ACCEPTED July 17, 2020.

INCLUDE WHEN CITING DOI: 10.3171/2020.7.FOCUS20418. 
trapolations from results obtained from biologically distinct tumors in younger populations. ${ }^{7-9}$

In this review, we provide a historical perspective on the successes and failures of the multimodal management of GBM in the elderly. We also report how progress on the genomic front has impacted what constitutes a maximum safe extent of resection (EOR) in the elderly, the choice and timing of chemoradiation, and the development of targeted therapy based on the unique genomic profiles of elderly GBM patients.

\section{Current Strategies for the Management of GBM in the Elderly Patient}

Standard of care for newly diagnosed GBM remains maximum safe resection followed by concurrent TMZ (75 $\mathrm{mg} / \mathrm{m}^{2} /$ day for 6 weeks) and external beam radiation therapy (EBRT; 60 Gy in 30 fractions over 6 weeks), with subsequent maintenance cycles $(\mathrm{n}=5)$ of TMZ $(150-200 \mathrm{mg}$ / $\mathrm{m}^{2} /$ day for the first 5 days of a 28 -day cycle). ${ }^{2}$ The adjuvant chemoradiation protocol is based on a clinical trial in patients younger than 70 years old with end data extracted to fit the elderly population. ${ }^{2}$ Few retrospective studies and prospective clinical trials have specifically addressed multimodal management of GBM in elderly cohorts (Table 1). ${ }^{9-24}$ Despite this research, the survival of elderly patients remains poor (Fig. 1).

Some elderly patients present with frailty, which is defined as loss of reserve (energy or cognitive function) and accumulation of deficits. ${ }^{25}$ Frailty has been shown to influence management decisions and predict surgical outcomes in elderly patients. ${ }^{25}$ Objectively, frailty is assessed using scales such as the Canadian Study of Health and Aging 70-item Frailty Index (CSHA-FI), based on history and physical examination (including comorbidities such as seizures, diabetes, and congestive heart failure). ${ }^{26} \mathrm{~A}$ simpler form of the CSHA-FI is the modified Frailty Index (mFI), including 11 items and shown to predict morbidity and mortality in elderly patients. ${ }^{26}$ In neurosurgery, frail patients (higher $\mathrm{mFI}$ ) or those with a poor Karnofsky Performance Status (KPS) have been less likely to undergo surgery $(\mathrm{p}=0.0002$, OR 0.15$)$ versus biopsy and have decreased overall survival (OS; $\mathrm{p}=0.0028) .{ }^{25}$

Even with a matched KPS and tumor size, however, age ( $\geq 65$ years) remains an independent risk factor for a poor prognosis. ${ }^{11}$ This suggests that elderly patients may have underlying differences in tumor biology and could benefit from a different management approach that includes assessing and targeting differences in underlying biology compared with that in younger patients. Below, we discuss the historical context for both surgical and medical management of newly diagnosed and recurrent GBM as it pertains to elderly patients.

\section{Surgery}

In GBM, a more aggressive EOR, such as lobectomy or supratotal resection (SupTR; resection of areas with FLAIR signal abnormality), has been shown to be superior to gross-total resection (GTR) in two studies (median OS 20.7 vs 15.5 months, respectively, $\mathrm{p}=0.001 ;{ }^{27}$ and median OS 44.1 vs 18.7 months, $\left.p=0.040^{28}\right)$. Unfortunately, these studies did not specifically address whether SupTR would benefit elderly patients by improving OS while maintaining baseline functional status. In the elderly, GTR and subtotal resection (STR) have been shown to be superior to partial resection (PR) or biopsy (median OS: GTR 17.7 vs STR 16.1 vs PR 11.4 vs biopsy 4 months). ${ }^{12}$ However, the EOR maximizing OS in the elderly patient, while preserving baseline quality of life, remains undefined.

To develop a new roadmap for maximal safe resection across all demographics, some authors conducted a retrospective multicenter cohort study of 761 GBM patients to assess the association of EOR of contrast-enhancing and nonenhancing masses with molecular and clinical information, including age ( $\geq 18$ years). ${ }^{29}$ This research demonstrated that resection of both contrast- and noncontrast-enhancing masses (SupTR) in younger patients (age $<65$ years) with isocitrate dehydrogenase 1/2 (IDHI/2)wildtype tumors resulted in survival outcomes similar to those of patients with $I D H 1 / 2$-mutant tumors (median OS 37.3 months). Interestingly, elderly patients (age $>65$ years) with $I D H 1 / 2$-wildtype tumors benefited the most from resection of only the contrast-enhancing mass (GTR, median OS 12.4 months). These findings suggest that in younger patients (regardless of IDHI/2 status), a SupTR is needed to provide the best survival outcome possible, while elderly patients benefit most from GTR with the preservation of baseline quality of life.

In retrospective studies, the benefit of surgery for recurrent GBM remains controversial at any age. ${ }^{30,31}$ Therefore, until prospective randomized trials are performed, the decision to reoperate should be based on clinical status and focal disease, with stereotactic radiosurgery (SRS) as a salvage option in patients too frail to undergo surgery. ${ }^{32}$ Looking ahead, we anticipate that genomics-based patient stratification (such as IDHI/2 status ${ }^{29}$ ) will contribute to the standardization of surgical management across age demographics and that optimizing the postresection adjuvant treatment regimen (chemotherapy and radiation) in the elderly cohort will be critical for targeting residual microscopic tumor foci left behind following GTR.

\section{Chemotherapy}

Prior to TMZ-based chemotherapy for GBM, the relevance of adjuvant chemotherapy for GBM was extremely controversial given mixed results from clinical trials. ${ }^{2,33}$ Before TMZ-based chemotherapy became part of the GBM standard of care, we performed a meta-analysis of 16 randomized clinical trials ( $>3000$ patients) to compare the survival benefit of radiation therapy (RT) alone versus that with RT plus multiple chemotherapeutic agents for high-grade glioma. ${ }^{4}$ This meta-analysis demonstrated that chemotherapy provided a significant survival benefit when combined with radiation. Given the significant impact of age on survival, we also evaluated age as a potential confounder of the positive effect of chemotherapy on survival. The vast majority of studies (14/16) were balanced in terms of age distribution across treatment arms. Of the two studies reporting an age imbalance, one showed $62 \%$ of patients younger than 50 years in the chemotherapy arm and $34 \%$ of patients younger than 50 in the radiation arm. ${ }^{34}$ The other study reported the opposite, with $48 \%$ of 
TABLE 1. Studies including elderly patients with GBM

\begin{tabular}{|c|c|c|c|c|c|c|}
\hline Authors \& Year & Study Design & $\begin{array}{c}\text { Publication } \\
\text { Year }\end{array}$ & $\begin{array}{l}\text { Age } \\
\text { (yrs) }\end{array}$ & Arms & $\begin{array}{l}\text { No. of } \\
\text { Cases }\end{array}$ & $\begin{array}{l}\text { Median OS } \\
\quad(\text { mos })\end{array}$ \\
\hline \multicolumn{7}{|l|}{ Surgery } \\
\hline Vuorinen et al., $2003^{9}$ & Prospective & 2003 & $\geq 65$ & $\begin{array}{l}\text { Biopsy } \\
\text { Resection }\end{array}$ & $\begin{array}{l}16 \\
14\end{array}$ & $\begin{array}{l}2.8 \\
5.6^{*}\end{array}$ \\
\hline Iwamoto et al., $2009^{10}$ & Retrospective & 2009 & $\geq 65$ & $\begin{array}{c}\text { GTR } \\
\text { PR } \\
\text { Biopsy }\end{array}$ & $\begin{array}{r}109 \\
212 \\
73\end{array}$ & \\
\hline Chaichana et al., $2011^{11}$ & Retrospective & 2011 & $\geq 65$ & $\begin{array}{l}\text { Resection } \\
\text { Biopsy }\end{array}$ & $\begin{array}{l}40 \\
40\end{array}$ & $\begin{array}{l}5.7^{*} \\
4.0\end{array}$ \\
\hline Oszvald et al., $2012^{12}$ & Retrospective & 2012 & $\geq 65$ & $\begin{array}{l}\text { GTR } \\
\text { STR } \\
\text { PR } \\
\text { Biopsy }\end{array}$ & $\begin{array}{l}19 \\
26 \\
35 \\
66\end{array}$ & $\begin{array}{c}17.7^{*} \\
16.1^{*} \\
11.4^{*} \\
4\end{array}$ \\
\hline \multicolumn{7}{|l|}{ Chemotherapy } \\
\hline Pérez-Larraya et al., $2011^{13}$ & Prospective phase 2 & 2011 & $\geq 70$ & TMZ & 70 & 6.2 \\
\hline Wick et al., $2013^{38}$ (NOA-08) & Prospective phase 3 & 2012 & $>60$ & $\begin{array}{c}\text { TMZ } \\
\text { Standard RT }\end{array}$ & $\begin{array}{l}195 \\
178\end{array}$ & $\begin{array}{l}8.2 \\
9.8\end{array}$ \\
\hline Malmström et al., $2012^{15}$ (NCBTSG) & Prospective phase 3 & 2012 & $>60$ & $\begin{array}{c}\text { Standard RT } \\
\text { Hypo-RT } \\
\text { TMZ }\end{array}$ & $\begin{array}{l}100 \\
123 \\
119\end{array}$ & $\begin{array}{l}6 \\
7.5 \\
8\end{array}$ \\
\hline \multicolumn{7}{|l|}{ RT } \\
\hline Keime-Guibert et al., $2007^{16}$ (ANOCEF) & Prospective randomized & 2007 & $\geq 70$ & $\begin{array}{l}\text { RT + supp care } \\
\text { Supp care alone }\end{array}$ & $\begin{array}{l}39 \\
42\end{array}$ & $\begin{array}{l}7.3 \\
4.2\end{array}$ \\
\hline Scott et al., $2011^{17}$ & Retrospective (registry) & 2011 & $>70$ & $\begin{array}{l}\text { RT } \\
\text { No RT }\end{array}$ & $\begin{array}{l}1817 \\
1019\end{array}$ & \\
\hline \multicolumn{7}{|l|}{ Hypo-RT vs standard RT } \\
\hline Bauman et al., $1994^{18}$ & Prospective & 1994 & $\geq 65$ & $\begin{array}{c}\text { Hypo-RT } \\
\text { Supp care (historical) } \\
\text { Standard RT (historical) }\end{array}$ & 29 & $\begin{array}{r}6^{*} \\
1 \\
10\end{array}$ \\
\hline Roa et al., $2004^{19}$ & Randomized phase 2 & 2004 & $\geq 65$ & $\begin{array}{c}\text { Standard RT } \\
\text { Hypo-RT }\end{array}$ & $\begin{array}{l}51 \\
49\end{array}$ & $\begin{array}{l}5.1 \\
5.6\end{array}$ \\
\hline Perry et al., $2017^{20}$ (CCRTG CE.6/EORTC) & Prospective randomized & 2017 & $\geq 65$ & $\begin{array}{c}\text { Hypo-RT } \\
\text { Hypo-RT w/ TMZ }\end{array}$ & $\begin{array}{l}281 \\
281\end{array}$ & $\begin{array}{l}7.6 \\
9.3\end{array}$ \\
\hline \multicolumn{7}{|l|}{ Standard RT vs concurrent RT w/ TMZ } \\
\hline Gerstein et al., $2010^{21}$ & Retrospective & 2010 & $\geq 65$ & Concurrent RT w/ TMZ & 51 & 11.5 \\
\hline Minniti et al., $2012^{22}$ & Prospective & 2012 & $\geq 70$ & Concurrent RT w/ TMZ & 32 & 10.6 \\
\hline \multicolumn{7}{|l|}{ Concurrent hypo-RT w/ TMZ } \\
\hline Minniti et al., $2008^{23}$ & Prospective & 2008 & $\geq 70$ & Concurrent hypo-RT w/ TMZ & 71 & 12.4 \\
\hline Cao et al., $2012^{24}$ & Retrospective & 2012 & $\geq 60$ & $\begin{array}{c}\text { Hypo-RT } \\
\text { Concurrent hypo-RT w/ TMZ }\end{array}$ & $\begin{array}{l}57 \\
55\end{array}$ & $\begin{array}{l}6.9 \\
9.3\end{array}$ \\
\hline
\end{tabular}

hypo-RT = hypofractionated RT; supp = supportive.

* Statistically significant.

patients younger than 50 years in the chemotherapy arm compared with $57 \%$ of patients under the age of 50 in the radiation arm. ${ }^{35}$ We performed the meta-analysis with and without both of these studies and found no effect on the overall analysis. Although no treatment-specific age data were reported in most of the studies reviewed, this metaanalysis corroborated the beneficial effect of chemotherapy independent of age.

Twelve years later, a prospective, randomized, multicenter phase 3 clinical trial was undertaken by the Euro- pean Organisation for Research and Treatment of Cancer (EORTC) and the National Cancer Institute of Canada (NCIC) Clinical Trials Group to assess the efficacy of RT with and without TMZ-based chemotherapy. ${ }^{2}$ The study enrolled 573 patients aged 18-70 years and compared two groups: RT alone and RT with TMZ, an orally administered alkylating agent that readily crosses the blood-brain barrier (BBB), with a less toxic side effect profile relative to those of prior drugs in its class. In combination with RT, TMZ significantly extended the OS of GBM patients, par- 


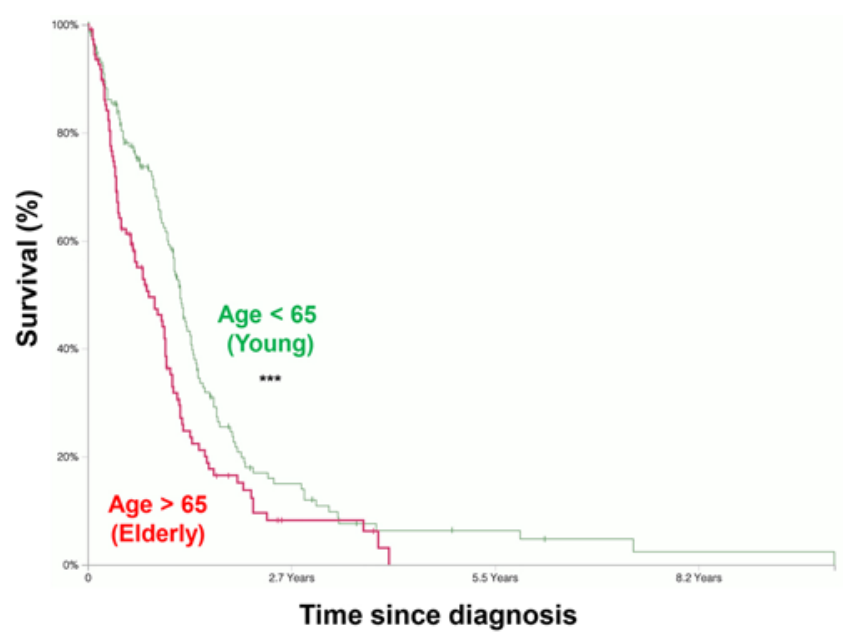

FIG. 1. Survival of patients with GBM based on age. Younger patients (age < 65 years, $n=147$ ) with IDH1/2-wildtype tumors have better survival than elderly patients (age $\geq 65$ years, $n=109$ ) with IDH1/2wildtype tumors. Data were obtained from The Cancer Genome Atlas (TCGA) and visualized on OncoScape. ${ }^{25}$ Given the significant impact of the IDH/2 mutation on survival, this analysis excluded all IDH1/2-mutant patients $(n=13) .{ }^{* * *} p<0.003$ (Cox proportional hazards regression).

ticularly for those presenting with methylation of the gene encoding the DNA repair enzyme, [6]-methylguanineDNA methyltransferase (MGMT; median OS 21.7 vs 15.3 months in unmethylated patients). A secondary endpoint analysis on health-related quality of life showed no detrimental effect following the addition of TMZ to RT. While some studies have shown a higher prevalence of $M G M T$ promoter hypermethylation in the elderly cohort, ${ }^{36,37}$ others have shown no significant difference across age demographics. ${ }^{38}$ Overall, these findings suggested that TMZbased chemotherapy was well tolerated and had an efficacy in elderly patients with newly diagnosed GBM similar to that in younger patients.

The DIRECTOR trial (Dose-Intensified Rechallenge With Temozolomide, One Week On One Week Off Versus Three Weeks On One Week Off in Patients With Progressive or Recurrent Glioblastoma trial) evaluated the efficacy of rechallenge with TMZ following GBM recurrence in patients aged 18-80 years. ${ }^{39} \mathrm{~A}$ multivariate analysis showed that MGMT promoter hypermethylation, but not age, predicted OS following treatment. Following TMZ reuse, the median time to treatment failure with $M G M T$ promoter hypermethylated tumors was 3.2 months versus 1.8 months in $M G M T$ promoter unmethylated tumors. Progression-free survival (PFS) rates at 6 months were $39.7 \%$ with $M G M T$ promoter hypermethylation and $5.9 \%$ without. Since this study included elderly patients whose age was $>65$ years, it suggests that TMZ rechallenge may also be effective and well tolerated by elderly patients with recurrent GBM.

\section{Radiation Therapy}

The relevance of RT for improving the OS of patients with GBM is well established based on randomized clinical trials. ${ }^{40,41}$ Elderly GBM patients (age $>70$ years) who undergo RT have improved OS compared to patients who do not undergo RT. ${ }^{17}$ Cognitive dysfunction and leukoencephalopathy, however, are significantly higher in elderly patients receiving standard RT courses. ${ }^{42,43}$ A prospective, phase 3 clinical trial (Nordic trial) randomized 291 patients 60 years of age or older to receive a standard 6-week course of RT (60 Gy administered in 2-Gy fractions over 6 weeks, $n=100)$, a shorter hypofractionated course of RT (34 Gy administered in 3.4-Gy fractions over 2 weeks, $\mathrm{n}=98)$, or TMZ alone $\left(200 \mathrm{mg} / \mathrm{m}^{2}\right.$ on days $1-5$ of every 28 days for up to 6 cycles, $\mathrm{n}=93) .{ }^{15}$ For patients with an age $>70$ years, OS was increased with TMZ (9 months, $\mathrm{p}<0.0001)$ and with hypofractionated RT $(7$ months, $\mathrm{p}$ $=0.02$ ) compared to that with standard RT (5.2 months). Patient compliance was also worse in the group assigned to the standard RT course than in the hypofractionated RT group ( $72 \%$ vs $94.9 \%$ treatment completion rate).

Given these findings, a prospective, randomized, phase 3 clinical trial was performed in elderly patients (age $\geq 65$ years) to assess the efficacy of a shorter course of RT (40 Gy in 15 fractions over 3 weeks) with or without concurrent TMZ ( $75 \mathrm{mg} / \mathrm{m}^{2} /$ day for 21 days), followed by adjuvant TMZ (150-200 mg/m²/day for 5 consecutive days of a 28-day cycle for up to 12 cycles or until disease progression); ${ }^{20} 562$ patients underwent randomization, and the median age was 73 years. Relative to a shorter course of RT alone, a shorter course of RT combined with TMZ was associated with a longer median PFS (5.3 vs 3.9 months, $\mathrm{p}<0.001)$ and median OS (9.3 vs 7.6 months, $\mathrm{p}<0.001)$. The effect was more pronounced in MGMT promoter hypermethylated GBM, with a median OS of 13.5 months versus 7.7 months with a shorter course of RT alone $(\mathrm{p}<$ 0.001). These findings indicate that a shorter course of RT combined with TMZ leads to improved OS compared to that with a shorter course of RT alone in elderly GBM patients. Toxicity was slightly higher in the group receiving a shorter course of RT with TMZ than in the group receiving the shorter course of RT alone. A baseline quality-oflife assessment for symptoms and function was performed and found to be similar between the two groups. Only nausea, vomiting, and constipation were associated with the time to deterioration (defined as a 10-point decrease in the score in function or symptoms). As expected, the time to deterioration was shorter in the combination therapy group relative to that in the group with the shorter-course RT. There were no other clinically important differences in the trial groups, indicating that the survival advantage was achieved without a significant impact on quality of life.

Some studies have shown a survival benefit with SRS for recurrent GBM. ${ }^{44}$ With an eye toward optimizing the response of recurrent $\mathrm{GBM}$ to $\mathrm{SRS}$, we retrospectively evaluated the pattern of treatment failure (failure location, extent of failure, and time to failure) in patients with recurrent GBM undergoing SRS at our institution over a 10 -year period..$^{32}$ We found that elderly patients presented with a more remote or out-of-field treatment failure location relative to that in the younger cohort (mean age for remote failure 68.3 years vs in-field failure 52.1 years, $p$ $=0.035$ ). Since $72 \%$ of our cohort was placed on bevacizumab (BEV; anti-vascular endothelial growth factor 
TABLE 2. Tumor genomic signatures of elderly GBM patients

\begin{tabular}{|c|c|c|c|}
\hline Authors \& Year & $\begin{array}{c}\text { Genomic \& } \\
\text { Epigenomic Status }\end{array}$ & $\begin{array}{l}\text { Proposed } \\
\text { Impact on } \\
\text { Prognosis }\end{array}$ & $\begin{array}{c}p \\
\text { Value* }\end{array}$ \\
\hline Ferguson et al., $2016^{36}$ & IDH1/2-wildtype & Poor & 0.0001 \\
\hline $\begin{array}{l}\text { Eckel-Passow et al., } \\
2015^{57}\end{array}$ & TERTp mutation & Poort & 0.0001 \\
\hline $\begin{array}{l}\text { Nghiemphu et al., } \\
200951\end{array}$ & $\begin{array}{c}\text { VEGF-A mRNA } \\
\text { upregulation }\end{array}$ & Poor & $<0.01$ \\
\hline $\begin{array}{l}\text { Cimino et al., } 2017^{72} \& \\
2018^{49} \\
\text { Fukai et al., } 2020^{67}\end{array}$ & $\begin{array}{l}\text { CDK4/MDM2 coampli- } \\
\text { fication }\end{array}$ & Poor & $\begin{array}{l}0.0121 \\
0.031 \ddagger\end{array}$ \\
\hline $\begin{array}{l}\text { Ferguson et al., } 2016^{36} \\
\text { Fukai et al., } 2020^{67}\end{array}$ & PTEN mutation & Poor & $\begin{array}{l}0.0258 \\
0.0404\end{array}$ \\
\hline Bozdag et al., $2013^{48}$ & $\begin{array}{l}\text { Higher integrin-medi- } \\
\text { ated cell migration } \\
\text { signature (migration } \\
\text { \& invasiveness) }\end{array}$ & Poor & $<0.03$ \\
\hline
\end{tabular}

* Difference between young and elderly GBM patients.

$\dagger$ In IDH1/2-wildtype patients.

$\ddagger$ CDK4 amplification only.

[VEGF] monoclonal antibody) following recurrence, we evaluated whether BEV use modified the effect of age on treatment failure location and found no significant effect (failure location OR 0.54, $\mathrm{p}=0.99$ ). There was also no significant association between age and extent of failure or time to failure. These findings indicate that elderly patients with focal recurrent GBM who are too frail to undergo resection may benefit from combining SRS with strategies that address microscopic disease, such as TMZbased chemotherapy, ${ }^{39}$ tumor-treating fields,${ }^{45}$ or immunotherapy. ${ }^{46}$

\section{Genomic Alterations and Adjuvant Treatment Options for the Elderly GBM Patient}

Most classic GBM-associated genomic alterations, such as inactivating TP53 mutations and EGFR amplification, are not over-represented in the elderly GBM cohort. ${ }^{47}$ However, tumors in elderly patients possess unique molecular signatures, including different genetic profiles (IDH1/2-wildtype status, telomerase reverse transcriptase promoter [TERTp] mutation, PTEN mutation), expression patterns (VEGF mRNA expression, higher integrin-mediated cell migration), and somatic copy number alterations (SCNAs; CDK4/MDM2 coamplification; Table 2). ${ }^{36,48-50}$ These biological differences provide opportunities for investigation of age-specific adjuvant treatment regimens.

\section{Vascular Endothelial Growth Factor A and Bevacizumab}

VEGF-A mRNA is upregulated in elderly patients with GBM..$^{50,51}$ The clinical relevance of this expression pattern was highlighted following the addition of BEV to TMZ-based chemoradiation regimens in patients with primary GBM in two studies, AVAglio (A Study of Bevacizumab [Avastin] in Combination With Temozolomide and Radiotherapy in Participants With Newly Diagnosed Glioblastoma) and RTOG 0825 (Temozolomide and Radiation Therapy With or Without Bevacizumab in Treating Patients With Newly Diagnosed Glioblastoma). ${ }^{52,53} \mathrm{Al}-$ though this regimen did not prolong OS in study cohorts, initial reports demonstrated preferential benefit in elderly patients (age $>50$ years vs younger patients with an age $<$ 50 years, $\mathrm{p}<0.005) .{ }^{54}$ Therefore, a phase 2 randomized clinical trial (ARTE [Avastin Plus Radiotherapy in Elderly Patients With Glioblastoma]) was conducted to evaluate the efficacy of BEV (10 mg/kg for 14 days) plus hypofractionated RT (40 Gy in 15 fractions; arm A, $\mathrm{n}=50$ ) versus hypofractionated RT alone ( $\operatorname{arm} \mathrm{B}, \mathrm{n}=25$ ) in elderly patients 65 years or older with GBM..$^{55}$ The median PFS was longer in arm A than in arm B (7.6 and 4.8 months, $\mathrm{p}=$ 0.003 ), but there was no significant difference in median OS between the two arms (12.1 and 12.2 months, respectively, $p=0.77)$. At the molecular level, prolonged PFS was restricted to tumors with receptor tyrosine kinase (RTK) I methylation subtype and a proneural gene expression profile. ${ }^{56}$ These findings suggest that underlying genomic signatures may confer susceptibility to targeted therapy especially in the elderly population. Additional clinical trials including elderly patients stratified based on these genomic features are needed to explore this finding. Today, even though BEV is not indicated for patients with newly diagnosed GBM, it remains FDA-approved for the treatment of $M G M T$ promoter unmethylated recurrent GBM and should be considered for elderly patients with tumor recurrence for the reduction of cerebral edema-associated symptoms and for its glucocorticoid-sparing effect.

\section{Metabolism: IDH1/2 Mutations}

Regardless of tumor grade or histology, IDHI/2 mutations are associated with significantly better OS and are prevalent in younger GBM patients but rare in elderly GBM patients. ${ }^{57}$ Approximately two-thirds of lower-grade gliomas, including all oligodendrogliomas and about half of all diffuse astrocytic gliomas, typically possess $I D H I / 2$ mutations; however, most GBMs lack $I D H 1 / 2$ mutations. ${ }^{58}$ In the 2016 WHO classification of central nervous system (CNS) tumors, IDH1/2-mutant and IDHI/2-wildtype tumors represent two distinct types of GBM..$^{59}$ This new designation is driven by the profoundly different biology and pathogenesis of IDH1/2-mutant compared to IDH1/2wildtype GBM. ${ }^{60}$ Given the unique biology and significantly worse outcomes in $I D H 1 / 2$-wildtype GBM patients, the cIMPACT-NOW (Consortium to Inform Molecular and Practical Approaches to CNS Tumor Taxonomy) members have proposed that future WHO CNS tumor classification schemes reserve the specific diagnosis of "GBM" for only IDH1/2-wildtype diffuse astrocytomas with high-grade histological or molecular features (WHO grade IV). ${ }^{60}$ These terminology changes, if incorporated into the upcoming CNS tumor classification, would have significant implications, as a GBM diagnosis will ultimately be more common in elderly patients purely based on the IDH1/2-wildtype status prevalence in this cohort.

Although the mechanism underlying survival differences between IDHI/2-wildtype and IDHI/2-mutant 


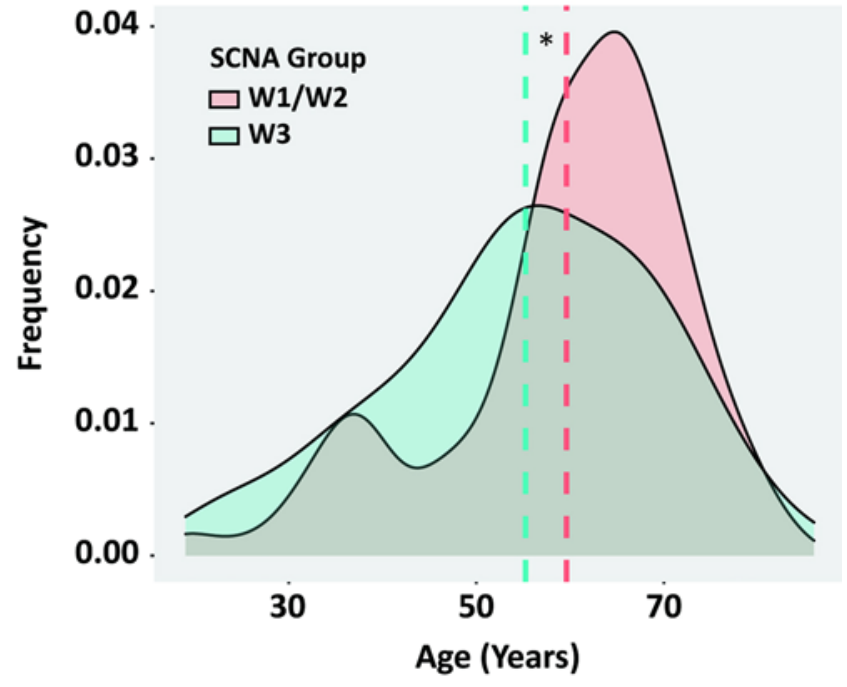

FIG. 2. SCNAs in GBM. Distribution shift of SCNA subtypes by age within the IDH1/2-wildtype GBM cohort from the GGN $(n=243)$. Distribution shows poor-prognosis SCNA subtypes (combined W1 and W2) occurring predominantly in the elderly population, while better-prognosis SCNA subtype W3 occurs more frequently in the younger population. Dashed lines represent means for SCNA subtypes W1/W2 (pink) and SCNA subtype W3 (green). * $p=0.0121$ (Mann-Whitney U-test, twotailed).

GBMs is still being elucidated, biologically relevant differences in tumor genetics, metabolism, and immune microenvironment have been identified ${ }^{61,62}$ While most studies have focused on targeting mutant $I D H 1 / 2$, preclinical evidence suggests that inhibiting $I D H 1 / 2$-wildtype activity in GBM could mimic the therapeutic response of IDH1/2-mutant tumors. ${ }^{63,64}$ Genetic and pharmacological inactivation of wildtype IDHI reduces levels of nicotinamide adenine dinucleotide phosphate (NADPH), which is critical for the biosynthesis of deoxynucleotides and antioxidants. This results in decreased cell growth, increases apoptosis following radiation-induced DNA damage, and prolongs the survival of mice bearing patient-derived xenografts. ${ }^{63,65}$ Human clinical trials are needed, but these findings suggest that targeting tumor metabolism by decreasing NADPH levels through wildtype $I D H I$ inhibition may be a therapeutic option for $I D H I$-wildtype tumors, prevalent in the elderly GBM cohort.

\section{Telomerase Activity: TERTp Mutations}

$T E R T$ encodes for the catalytic subunit of telomerase. Telomerase activity is typically restricted in human somatic cells, in which there are a finite number of cellular divisions prior to senescence and death. ${ }^{66}$ In tumor cells with unlimited replication potential, telomerase activity has been shown to be high. ${ }^{66}$ In GBM, gain-of-function TERTp mutations are more prevalent within IDHI/2-wildtype tumors and elderly patients. ${ }^{57,67}$ In elderly patients, TERTp mutations have been associated with a significantly lower OS than that in patients with wildtype TERTp tumors (10.4 vs 18 months, $\mathrm{p}=0.01){ }^{68}$ These findings suggest that targeting mutant TERTp may provide another therapeutic approach for elderly GBM patients. An ongoing phase 1/2 human vaccine clinical trial (NCT04280848, Anticancer Therapeutic Vaccination Using Telomerase-Derived Universal Cancer Peptides in Glioblastoma [UCPVax-Glio]) is evaluating the safety and efficacy of telomerase-derived universal cancer peptides in GBM following standard GBM treatment. ${ }^{69}$ Other ongoing strategies to target telomerase activity in GBM have shown some efficacy but remain in the preclinical stages of development, including small molecule-based inhibitors of telomerase activity such as ETP-47228, ETP-47037, and CRISPR-based editing of mutant TERTp..$^{70,71}$

\section{Somatic Copy Number Alterations: CDK4/MDM2 Coamplification}

There is growing evidence that SCNAs, present at both the gene and chromosomal levels, may provide prognostic information for patients with GBM. ${ }^{49,72,73}$ Utilizing a large GBM series, the German Glioma Network (GGN), we have demonstrated that SCNA subgroups (determined by differential status of coamplification of CDK4/MDM2, gain of chromosome 1, and gain of chromosome 19) provide prognostic information ${ }^{49,72}$ and show an increased frequency of subtypes associated with a worse prognosis (specifically $\mathrm{W} 1$ and $\mathrm{W} 2$ with $C D K 4 / M D M 2$ amplification) in the elderly population (Fig. 2). Another recent study of a Japanese cohort (Kansai Molecular Diagnosis Network for CNS tumors) compared SCNAs between elderly and younger $I D H 1 / 2$-wildtype GBM patients. ${ }^{67}$ Consistent with our findings, there was a higher prevalence of CDK4 amplification/gain in the elderly cohort. This suggests that these IDHI/2-wildtype GBM-associated SCNA signatures (CDK4/MDM2 amplification) may contribute to less favorable outcomes in the elderly population.

Large GBM data sets, such as the GGN and The Cancer Genome Atlas (TCGA), provide information about SCNA subtype distribution across general populations but do not address the potential biases present in clinical trial populations, which are biased toward including younger patients and/or those with a better functional status. For example, the randomized phase 2 ARTE clinical trial, which was focused on newly diagnosed GBM in the elderly population, ${ }^{55}$ showed that a majority of elderly patients enrolled in the study had a favorable SCNA signature, unlike the general GBM population, in which a majority of elderly patients have an unfavorable SCNA signature. ${ }^{49}$ This has several implications for GBM clinical trials in the elderly, including the need to balance molecular signatures across trial arms or the desirability of trials tailored toward SCNA subtype-specific responses. Therefore, it may be prudent to perform SCNA profiling at the time of clinical trial enrollment to account for such potential biases, and to facilitate identification of SCNA subtype-specific responses for elderly patients with GBM.

Therapeutically, selective cyclin-dependent kinase (CDK) inhibition may be an option for elderly cohortprevalent SCNA subtypes (W1 and W2) with CD4/MDM2 coamplification. Selective CDK4/6 inhibitors such as palbociclib, ribociclib, and abemaciclib have been in clinical trials for other cancers including breast cancer, with ribociclib now used as first-line therapy for advanced hormone receptor-positive (HR+) breast cancer ${ }^{74}$ In GBM, 
a phase 0 human clinical trial of ribociclib demonstrated excellent $\mathrm{BBB}$ penetration and distribution within tumors in patients, although there was limited clinical efficacy as monotherapy in recurrent GBM ${ }^{75}$ Therefore, additional clinical trials assessing the efficacy of ribociclib inhibitors as part of a combinatorial strategy with other therapies are indicated in GBM, particularly in the W1 and W2 SCNA subtype prevalent in the elderly cohort.

\section{Conclusions}

Elderly patients with GBM have a much poorer prognosis than younger patients with GBM. This difference in outcomes could be attributed to multiple factors, including a poorer baseline health status and age-related differences in underlying tumor biology. Currently, the best available evidence for the management of newly diagnosed GBM in elderly patients is resection of the contrast-enhancing lesion only ${ }^{29}$ and a shorter hypofractionated course of RT (40 Gy in 15 fractions over 3 weeks) in combination with standard concurrent and maintenance TMZ chemotherapy. ${ }^{20}$ This allows for higher treatment compliance and improves OS while maintaining a baseline quality of life. ${ }^{15}$ However, regardless of treatment (standard ${ }^{2}$ or modified ${ }^{20}$ ), survival outcomes in the elderly GBM cohort remain dismal. Major prognostic and biological differences driving the pathogenesis and natural history of GBM in the elderly compared with younger patients appear to be genomic. We propose that future surgical and medical clinical trials be agnostic to age and designed to stratify patients based on biological and clinically important genomic characteristics.

\section{Acknowledgments}

We wish to thank Sharon Durfy, $\mathrm{PhD}$, for her assistance with manuscript preparation.

Funding was provided by the National Institute of Neurological Disorders and Stroke, National Institutes of Health grant no. R25NS079200 (C.I.E.).

\section{References}

1. Ostrom QT, Gittleman H, Liao P, et al. CBTRUS Statistical Report: primary brain and other central nervous system tumors diagnosed in the United States in 2010-2014. Neuro Oncol.2017;19(suppl_5):v1-v88.

2. Stupp R, Mason WP, van den Bent MJ, et al. Radiotherapy plus concomitant and adjuvant temozolomide for glioblastoma. N Engl J Med. 2005;352(10):987-996.

3. Laperriere N, Weller M, Stupp R, et al. Optimal management of elderly patients with glioblastoma. Cancer Treat Rev. 2013; 39(4):350-357.

4. Fine HA, Dear KB, Loeffler JS, et al. Meta-analysis of radiation therapy with and without adjuvant chemotherapy for malignant gliomas in adults. Cancer. 1993;71(8):2585-2597.

5. Hess KR, Broglio KR, Bondy ML. Adult glioma incidence trends in the United States, 1977-2000. Cancer. 2004; 101(10):2293-2299.

6. Ostrom QT, Gittleman H, Farah P, et al. CBTRUS Statistical Report: primary brain and central nervous system tumors diagnosed in the United States in 2006-2010. Neuro Oncol. 2013;15(suppl 2):ii1-ii56.

7. Ostrom QT, Gittleman H, Truitt G, et al. CBTRUS Statistical Report: primary brain and other central nervous system tumors diagnosed in the United States in 2011-2015. Neuro Oncol. 2018;20(suppl_4):iv1-iv86.

8. Iwamoto FM, Reiner AS, Nayak L, et al. Prognosis and patterns of care in elderly patients with glioma. Cancer. 2009; 115(23):5534-5540.

9. Vuorinen V, Hinkka S, Färkkilä M, Jääskeläinen J. Debulking or biopsy of malignant glioma in elderly people-a randomised study. Acta Neurochir (Wien). 2003;145(1):5-10.

10. Iwamoto FM, Cooper AR, Reiner AS, et al. Glioblastoma in the elderly: the Memorial Sloan-Kettering Cancer Center experience (1997-2007). Cancer. 2009;115(16):3758-3766.

11. Chaichana KL, Garzon-Muvdi T, Parker S, et al. Supratentorial glioblastoma multiforme: the role of surgical resection versus biopsy among older patients. Ann Surg Oncol. 2011; 18(1):239-245.

12. Oszvald A, Güresir E, Setzer M, et al. Glioblastoma therapy in the elderly and the importance of the extent of resection regardless of age. J Neurosurg. 2012;116(2):357-364.

13. Pérez-Larraya J, Ducray F, Chinot O, et al. Temozolomide in elderly patients with newly diagnosed glioblastoma and poor performance status: an ANOCEF phase II trial. J Clin Oncol. 2011;29(22):3050-3055.

14. Wick W, Platten M, Meisner C, et al. Temozolomide chemotherapy alone versus radiotherapy alone for malignant astrocytoma in the elderly: the NOA-08 randomised, phase 3 trial. Lancet Oncol. 2012;13(7):707-715.

15. Malmström A, Grønberg BH, Marosi C, et al. Temozolomide versus standard 6-week radiotherapy versus hypofractionated radiotherapy in patients older than 60 years with glioblastoma: the Nordic randomised, phase 3 trial. Lancet Oncol. 2012;13(9):916-926.

16. Keime-Guibert F, Chinot O, Taillandier L, et al. Radiotherapy for glioblastoma in the elderly. N Engl J Med. 2007; 356(15):1527-1535.

17. Scott J, Tsai YY, Chinnaiyan P, Yu HH. Effectiveness of radiotherapy for elderly patients with glioblastoma. Int J Radiat Oncol Biol Phys. 2011;81(1):206-210.

18. Bauman GS, Gaspar LE, Fisher BJ, et al. A prospective study of short-course radiotherapy in poor prognosis glioblastoma multiforme. Int J Radiat Oncol Biol Phys. 1994;29(4):835839.

19. Roa W, Brasher PM, Bauman G, et al. Abbreviated course of radiation therapy in older patients with glioblastoma multiforme: a prospective randomized clinical trial. J Clin Oncol. 2004;22(9):1583-1588.

20. Perry JR, Laperriere N, O'Callaghan CJ, et al. Short-course radiation plus temozolomide in elderly patients with glioblastoma. N Engl J Med. 2017;376(11):1027-1037.

21. Gerstein J, Franz K, Steinbach JP, et al. Postoperative radiotherapy and concomitant temozolomide for elderly patients with glioblastoma. Radiother Oncol. 2010;97(3):382-386.

22. Minniti G, Lanzetta G, Scaringi C, et al. Phase II study of short-course radiotherapy plus concomitant and adjuvant temozolomide in elderly patients with glioblastoma. Int $J$ Radiat Oncol Biol Phys. 2012;83(1):93-99.

23. Minniti G, De Sanctis V, Muni R, et al. Radiotherapy plus concomitant and adjuvant temozolomide for glioblastoma in elderly patients. J Neurooncol. 2008;88(1):97-103.

24. Cao JQ, Fisher BJ, Bauman GS, et al. Hypofractionated radiotherapy with or without concurrent temozolomide in elderly patients with glioblastoma multiforme: a review of ten-year single institutional experience. J Neurooncol. 2012; 107(2):395-405.

25. Cloney M, D'Amico R, Lebovic J, et al. Frailty in geriatric glioblastoma patients: a predictor of operative morbidity and outcome. World Neurosurg. 2016;89:362-367.

26. Tsiouris A, Hammoud ZT, Velanovich V, et al. A modified frailty index to assess morbidity and mortality after lobectomy. J Surg Res. 2013;183(1):40-46. 
27. Li YM, Suki D, Hess K, Sawaya R. The influence of maximum safe resection of glioblastoma on survival in 1229 patients: can we do better than gross-total resection? J Neurosurg. 2016;124(4):977-988.

28. Roh TH, Kang SG, Moon JH, et al. Survival benefit of lobectomy over gross-total resection without lobectomy in cases of glioblastoma in the noneloquent area: a retrospective study. $J$ Neurosurg. 2020;132(3):895-901.

29. Molinaro AM, Hervey-Jumper S, Morshed RA, et al. Association of maximal extent of resection of contrast-enhanced and non-contrast-enhanced tumor with survival within molecular subgroups of patients with newly diagnosed glioblastoma. JAMA Oncol. 2020;6(4):495-503.

30. Gorlia T, Stupp R, Brandes AA, et al. New prognostic factors and calculators for outcome prediction in patients with recurrent glioblastoma: a pooled analysis of EORTC Brain Tumour Group phase I and II clinical trials. Eur J Cancer. 2012;48(8):1176-1184.

31. Suchorska B, Weller M, Tabatabai G, et al. Complete resection of contrast-enhancing tumor volume is associated with improved survival in recurrent glioblastoma-results from the DIRECTOR trial. Neuro Oncol. 2016;18(4):549-556.

32. Ene CI, Macomber MW, Barber JK, et al. Patterns of failure after stereotactic radiosurgery for recurrent high-grade glioma: a single institution experience of 10 years. Neurosurgery. 2019;85(2):E322-E331.

33. Chamberlain MC. Treatment options for glioblastoma. Neurosurg Focus. 2006;20(4):E19.

34. Garrett MJ, Hughes HJ, Freedman LS. A comparison of radiotherapy alone with radiotherapy and CCNU in cerebral glioma. Clin Oncol. 1978;4(1):71-76.

35. Trojanowski T, Peszyński J, Turowski K, et al. Postoperative radiotherapy and radiotherapy combined with CCNU chemotherapy for treatment of brain gliomas. J Neurooncol. 1988; 6(3):285-291.

36. Ferguson SD, Xiu J, Weathers SP, et al. GBM-associated mutations and altered protein expression are more common in young patients. Oncotarget. 2016;7(43):69466-69478.

37. Gerstner ER, Yip S, Wang DL, et al. MGMT methylation is a prognostic biomarker in elderly patients with newly diagnosed glioblastoma. Neurology. 2009;73(18):1509-1510.

38. Wick W, Meisner C, Hentschel B, et al. Prognostic or predictive value of MGMT promoter methylation in gliomas depends on IDH1 mutation. Neurology. 2013;81(17):1515-1522.

39. Weller M, Tabatabai G, Kästner B, et al. MGMT promoter methylation is a strong prognostic biomarker for benefit from dose-intensified temozolomide rechallenge in progressive glioblastoma: the DIRECTOR trial. Clin Cancer Res. 2015; 21(9):2057-2064.

40. Andersen AP. Postoperative irradiation of glioblastomas. Results in a randomized series. Acta Radiol Oncol Radiat Phys Biol. 1978;17(6):475-484.

41. Sandberg-Wollheim M, Malmström P, Strömblad LG, et al. A randomized study of chemotherapy with procarbazine, vincristine, and lomustine with and without radiation therapy for astrocytoma grades 3 and/or 4. Cancer. 1991;68(1):22-29.

42. Saito K, Mukasa A, Narita Y, et al. Toxicity and outcome of radiotherapy with concomitant and adjuvant temozolomide in elderly patients with glioblastoma: a retrospective study. Neurol Med Chir (Tokyo). 2014;54(4):272-279.

43. Brandes AA, Franceschi E, Tosoni A, et al. Temozolomide concomitant and adjuvant to radiotherapy in elderly patients with glioblastoma: correlation with MGMT promoter methylation status. Cancer. 2009;115(15):3512-3518.

44. Romanelli P, Conti A, Pontoriero A, et al. Role of stereotactic radiosurgery and fractionated stereotactic radiotherapy for the treatment of recurrent glioblastoma multiforme. Neurosurg Focus. 2009;27(6):E8.

45. Stupp R, Taillibert S, Kanner A, et al. Effect of tumor-treat- ing fields plus maintenance temozolomide vs maintenance temozolomide alone on survival in patients with glioblastoma: a randomized clinical trial. JAMA. 2017;318(23):2306-2316.

46. Reardon DA, Dietrich J, Clarke JL, et al. Phase II study to evaluate safety and efficacy of MEDI4736 (durvalumab) + radiotherapy in patients with newly diagnosed unmethylated MGMT glioblastoma (new unmeth GBM). J Clin Oncol. 2019;37(15 suppl):2032.

47. Li G, Zhai Y, Wang Z, et al. Postoperative standard chemoradiotherapy benefits primary glioblastoma patients of all ages. Cancer Med. 2020;9(6):1955-1965.

48. Bozdag S, Li A, Riddick G, et al. Age-specific signatures of glioblastoma at the genomic, genetic, and epigenetic levels. PLoS One. 2013;8(4):e62982.

49. Cimino PJ, McFerrin L, Wirsching HG, et al. Copy number profiling across glioblastoma populations has implications for clinical trial design. Neuro Oncol. 2018;20(10):1368-1373.

50. Lee Y, Scheck AC, Cloughesy TF, et al. Gene expression analysis of glioblastomas identifies the major molecular basis for the prognostic benefit of younger age. BMC Med Genomics. 2008;1:52.

51. Nghiemphu PL, Liu W, Lee Y, et al. Bevacizumab and chemotherapy for recurrent glioblastoma: a single-institution experience. Neurology. 2009;72(14):1217-1222.

52. Chinot OL, Wick W, Mason W, et al. Bevacizumab plus radiotherapy-temozolomide for newly diagnosed glioblastoma. N Engl J Med. 2014;370(8):709-722.

53. Gilbert MR, Dignam JJ, Armstrong TS, et al. A randomized trial of bevacizumab for newly diagnosed glioblastoma. $N$ Engl J Med. 2014;370(8):699-708.

54. Lai A, Tran A, Nghiemphu PL, et al. Phase II study of bevacizumab plus temozolomide during and after radiation therapy for patients with newly diagnosed glioblastoma multiforme. J Clin Oncol. 2011;29(2):142-148.

55. Wirsching HG, Tabatabai G, Roelcke U, et al. Bevacizumab plus hypofractionated radiotherapy versus radiotherapy alone in elderly patients with glioblastoma: the randomized, open-label, phase II ARTE trial. Ann Oncol. 2018;29(6): 1423-1430.

56. Snuderl M, Fazlollahi L, Le LP, et al. Mosaic amplification of multiple receptor tyrosine kinase genes in glioblastoma. Cancer Cell. 2011;20(6):810-817.

57. Eckel-Passow JE, Lachance DH, Molinaro AM, et al. Glioma groups based on $1 \mathrm{p} / 19 \mathrm{q}$, IDH, and TERT promoter mutations in tumors. $N$ Engl J Med. 2015;372(26):2499-2508.

58. Yan H, Parsons DW, Jin G, et al. IDH1 and IDH2 mutations in gliomas. N Engl J Med. 2009;360(8):765-773.

59. Louis DN, Perry A, Reifenberger G, et al. The 2016 World Health Organization Classification of Tumors of the Central Nervous System: a summary. Acta Neuropathol. 2016;131(6): 803-820.

60. Brat DJ, Aldape K, Colman H, et al. cIMPACT-NOW update 5: recommended grading criteria and terminologies for IDH-mutant astrocytomas. Acta Neuropathol. 2020;139(3): 603-608.

61. Amankulor NM, Kim Y, Arora S, et al. Mutant IDH1 regulates the tumor-associated immune system in gliomas. Genes Dev. 2017:31(8):774-786.

62. Kohanbash G, Carrera DA, Shrivastav S, et al. Isocitrate dehydrogenase mutations suppress STAT1 and CD8+ T cell accumulation in gliomas. J Clin Invest. 2017;127(4):1425-1437.

63. Wahl DR, Dresser J, Wilder-Romans K, et al. Glioblastoma therapy can be augmented by targeting IDH1-mediated NADPH biosynthesis. Cancer Res. 2017;77(4):960-970.

64. Molenaar RJ, Maciejewski JP, Wilmink JW, van Noorden CJF. Wild-type and mutated IDH1/2 enzymes and therapy responses. Oncogene. 2018;37(15):1949-1960.

65. Zarei M, Lal S, Parker SJ, et al. Posttranscriptional upregulation of IDH1 by HuR establishes a powerful survival phe- 
notype in pancreatic cancer cells. Cancer Res. 2017;77(16): 4460-4471.

66. Zvereva MI, Shcherbakova DM, Dontsova OA. Telomerase: structure, functions, and activity regulation. Biochemistry (Mosc). 2010;75(13):1563-1583.

67. Fukai J, Arita H, Umehara T, et al. Molecular characteristics and clinical outcomes of elderly patients with IDH-wildtype glioblastomas: comparative study of older and younger cases in Kansai Network cohort. Brain Tumor Pathol. 2020;37(2): $50-59$.

68. Sasaki T, Fukai J, Kodama Y, et al. Characteristics and outcomes of elderly patients with diffuse gliomas: a multi-institutional cohort study by Kansai Molecular Diagnosis Network for CNS Tumors. J Neurooncol. 2018;140(2):329-339.

69. Centre Hospitalier Universitaire de Besancon. Anticancer therapeutic vaccination using telomerase-derived universal cancer peptides in glioblastoma (UCPVaxGlio). NCT04280848. Accessed August 17, 2020. https:// clinicaltrials.gov/ct2/show/NCT04280848

70. Bejarano L, Schuhmacher AJ, Méndez M, et al. Inhibition of TRF1 telomere protein impairs tumor initiation and progression in glioblastoma mouse models and patient-derived xenografts. Cancer Cell. 2017;32(5):590-607.e4.

71. Li X, Qian X, Wang B, et al. Programmable base editing of mutated TERT promoter inhibits brain tumour growth. Nat Cell Biol. 2020;22(3):282-288.

72. Cimino PJ, Zager M, McFerrin L, et al. Multidimensional scaling of diffuse gliomas: application to the 2016 World Health Organization classification system with prognostically relevant molecular subtype discovery. Acta Neuropathol Commun. 2017:5(1):39.

73. Umehara T, Arita H, Yoshioka E, et al. Distribution differ- ences in prognostic copy number alteration profiles in IDHwild-type glioblastoma cause survival discrepancies across cohorts. Acta Neuropathol Commun. 2019;7(1):99.

74. Hortobagyi GN, Stemmer SM, Burris HA, et al. Ribociclib as first-line therapy for HR-positive, advanced breast cancer. $N$ Engl J Med. 2016;375(18):1738-1748.

75. Tien AC, Li J, Bao X, et al. A phase 0 trial of ribociclib in recurrent glioblastoma patients incorporating a tumor pharmacodynamic- and pharmacokinetic-guided expansion cohort. Clin Cancer Res. 2019;25(19):5777-5786.

\section{Disclosures}

The authors report no conflict of interest concerning the materials or methods used in this study or the findings specified in this paper.

\section{Author Contributions}

Conception and design: Ene, Cimino. Analysis and interpretation of data: all authors. Drafting the article: all authors. Critically revising the article: all authors. Reviewed submitted version of manuscript: all authors. Approved the final version of the manuscript on behalf of all authors: Ene. Study supervision: Fine, Holland.

\section{Correspondence}

Chibawanye I. Ene: University of Washington School of Medicine, Seattle,WA.respub@uw.edu. 